\title{
Conhecimento de cirurgiões-dentistas sobre o diagnóstico e o tratamento de lesões cervicais não cariosas: um estudo piloto em rede colaborativa
}

\author{
Knowledge of dental-surgeons regarding diagnosis and treatment of \\ non-carious cervical lesions: a pilot study in collaborative network
}

\author{
Rodrigo Antonio Modena* \\ Abner Fernando Senna Pires* \\ Patrícia Nivoloni Tannure ${ }^{* * *}$ \\ Larissa Maria Assad Cavalcante $e^{* * * *}$ \\ Luis Felipe Jochims Schneider ${ }^{* * * *}$
}

\section{Resumo}

Objetivo: identificar o perfil de formação e atendimento de cirurgiões-dentistas (CDs) e procurar compreender qual a conduta prestada por esses profissionais em relação ao diagnóstico e ao tratamento das lesões cervicais não cariosas (LCNCS). Sujeitos e método: um questionário foi disponibilizado e enviado para CDs com o cadastro atualizado na base de dados do Conselho Regional de Odontologia do Rio de Janeiro. Os resultados foram analisados de forma descritiva e o teste de Qui-Quadrado com nível de significância de 5\%. Resultados: a maioria dos CDs procura fazer distinção entre as diferentes formas de LCNCs $(76,5 \%)$, porém não usa método auxiliar (83,2\%) e não possui questionário específico $(59,7 \%)$. Para $40,3 \%$ dos CDs, a etiologia das LCNCs é unifatorial ou não soube responder. A maioria faz uso de isolamento relativo $(65,6 \%)$ e utiliza técnicas de retração/afastamento gengival (83,2\%). O sistema adesivo convencional de dois passos (63\%) e a resina composta do tipo híbrida/micro-híbrida $(52,1 \%)$ são os materiais mais utilizados. Com relação à longevidade das restaurações, apenas $17,6 \%$ dos CDs acreditam na duração por um período acima de 5 anos. Com relação às associações, houve relação entre a forma de remuneração e o uso/método de isolamento $(p=0,004)$ e entre a forma de remuneração e o uso de técnicas de retração/afastamento gengival ( $p=0,005)$. Conclusões: os CDs não possuem um protocolo preestabelecido para correto diagnóstico de LCNCs. A forma de remuneração influencia no uso do isolamento para o tratamento restaurador. O sistema adesivo convencional de dois passos é o mais utilizado pelos CDs.

Palavras-chave: Abfração. Abrasão. Biocorrosão. Erosão. Lesões cervicais não cariosas.

\section{Introdução}

As lesões cervicais não cariosas (LCNCs) são caracterizadas pela perda de estrutura dental nas proximidades da junção cemento-esmalte (JCE) por meio de um processo não carioso ${ }^{1,2}$. Enquanto a redução da atividade de cárie é uma realidade em determinadas populações ${ }^{3}$, nota-se um aumento considerável na prática odontológica de problemas relacionados às $\mathrm{LCNCs}^{1,3,4}$. A prevalência desse tipo de lesão tem sido relatada como sendo de $5 \%$ a $85 \%$, em vários estudos populacionais ${ }^{5-7}$. Esse fato pode

Graduando em Odontologia, Faculdade de Odontologia da Universidade Federal Fluminense, Niterói, RJ, Brasil.

Mestrando (profissional) em Odontologia,Universidade Veiga de Almeida. Professor do curso de graduação em Odontologia da Universidade Veiga de Almeida, Rio de Janeiro, RJ, Brasil.

Doutora e mestre em Odontopediatria, Universidade Federal do Rio de Janeiro. Professora do Mestrado Profissional em Odontologia da Universidade Veiga de Almeida, Rio de Janeiro, RJ, Brasil.

Doutora e mestre em Clínica Odontológica, Faculdade de Odontologia de Piracicaba, Universidade Estadual de Campinas. Professora do Mestrado Profissional em Odontologia da Universidade Veiga de Almeida, Rio de Janeiro, RJ, Brasil. Professora adjunta da Faculdade de Odontologia da Universidade Federal Fluminense, Niterói, RJ, Brasil. Professora do curso de Odontologia da Universidade Salgado de Oliveira, Niterói, RJ, Brasil.

***** Doutor e mestre em Materiais Dentários, Faculdade de Odontologia de Piracicaba, Universidade de Campinas. Professor e coordenador adjunto do curso de Mestrado Profissional em Odontologia da Universidade Veiga de Almeida, Rio de Janeiro, RJ, Brasil. Professor adjunto da Faculdade de Odontologia da Universidade Federal Fluminense, Niterói, RJ, Brasil. 
ser explicado pelo aumento da expectativa de vida da população, pela manutenção da dentição natural em idade avançada e pelo aumento da exposição dos dentes aos fatores etiológicos relacionados às $\mathrm{LCNCs}^{1,8}$. De forma mais específica, as LCNCs podem ser resultantes dos processos de abfração, abrasão e erosão (biocorrosão), bem como suas interações ${ }^{6,9,10}$.

A abfração é a perda patológica de estrutura dental por meio de forças biomecânicas excessivas, resultando em flexão com falha de esmalte na região cervical dos dentes e apresenta-se em forma de cunha $^{11}$. A abrasão decorre do desgaste patológico do dente por meio de processos mecânicos repetitivos, e as lesões assumem forma de V, tendo aspecto liso e brilhante ${ }^{11,12}$. O termo "biocorrosão" está sendo utilizado para denominar as lesões por erosão e caracteriza-se pela ação bioquímica ou eletroquímica que provoca a degradação molecular das propriedades essenciais em um tecido vivo ${ }^{6,9,10}$. Basicamente, a biocorrosão pode ocorrer por meio de ação química exógena ou bioquímica endógena, como enzimas proteolíticas bioquímicas e por efeito piezoelétrico ${ }^{13}$. Normalmente, esse tipo de lesão é caracterizada na forma de pires, com perda do brilho dos dentes e provável exposição dentinária ${ }^{14}$.

A etiologia das LCNCs é controversa na literatura $^{15-17} \mathrm{e}$, devido à interação complexa de diversos mecanismos, a causa de determinada forma de lesão não é relacionada a um único mecanismo iso$\operatorname{lado}^{10}$, atribuindo às LCNCs um caráter multifatorial $^{6,8,10,18,19}$. As LCNCs estão mais presentes no sexo feminino, o grupo dos pré-molares são os dentes mais acometidos, em especial na face vestibular, e a abfração é o tipo mais prevalente dentre as lesões ${ }^{20}$.

Estudos prévios mostraram discordância entre os profissionais a respeito de causas, métodos de diagnóstico, tipos de tratamento, formas de prevenção assim como fatores associados a cada tipo de LCNC ${ }^{4,17,21}$. Assim, é fundamental o desenvolvimento de trabalhos que visem determinar as formas de diagnóstico e tratamento empregadas por cirurgiões-dentistas (CDs), bem como entender os fatores individuais que podem influenciar tanto a forma de diagnóstico quanto o tratamento restaurador de LCNCs.

Atualmente, sabe-se que, com relação à pesquisa voltada para a saúde humana, novas ferramentas são necessárias para que a disseminação do conhecimento ocorra de forma mais ágil e eficiente. Uma forma de agilizar a translação dos resultados de uma pesquisa é promover o envolvimento entre profissionais de pesquisa e profissionais que atuam diretamente na prática clínica, e é com essa finalidade que surgiram as redes de pesquisa baseadas na prática odontológica (RPBPOs, do inglês Practice-Based Research Network - PBRN). Essas redes colaborativas oferecem uma excelente oportunidade para que haja o envolvimento entre os profissionais de diversas áreas. Para aqueles que atuam diaria- mente na área clínica, pode ocorrer o envolvimento no processo de pesquisa e na formulação de questionamentos de investigação clínica que podem melhorar a saúde bucal da população e sua própria prática diária. Além disso, as RPBPOs oferecem aos pesquisadores a oportunidade de aprender mais sobre questões cotidianas que envolvem a saúde oral e interagir com profissionais da área odontológica ${ }^{22-25}$. Diante desse cenário, as RPBPOs têm um grande potencial de contribuição com dados para o modelo de odontologia baseada em evidências, definido pela American Dental Association como: "abordagem aos cuidados bucais que requer a integração judiciosa de: a) avaliações sistemáticas de evidências clínicas, cientificamente pertinentes, relativas à condição e à história médica e odontológica de pacientes; b) perícia clínica do dentista; c) necessidades e preferências do paciente" ${ }^{26}$.

Diante do exposto, as RPBPOs geram conhecimento baseado em evidências. Dessa forma, é evidente que a criação de grupos baseados na prática clínica é de fundamental importância para a melhora da saúde da população. Por essa razão, o objetivo do presente estudo foi identificar, por meio de uma rede de pesquisa colaborativa, o perfil de formação e atendimento dos cirurgiões-dentistas e procurar compreender qual a conduta prestada pelos CDs frente ao diagnóstico e ao tratamento das lesões cervicais não cariosas.

\section{Sujeitos e método}

O estudo foi aprovado pelo Comitê de Ética e Pesquisa da Universidade Federal Fluminense (Protocolo $\mathrm{n}^{\circ}$ 33723514.7.0000.5243). Para o presente estudo foi elaborado um questionário adaptado de Lyttle et al. ${ }^{21}$ (1998) e Gordan et al. ${ }^{27}$, baseado nos conceitos de Grippo et al. ${ }^{10}$ (2012). O questionário foi dividido em três grandes grupos: referente ao perfil de formação e atendimento dos CDs, ao perfil de diagnóstico das LCNCs e ao perfil do tratamento restaurador para as restaurações diretas de LCNCs (Tabelas 1, 2 e 3).

Foram incluídos no estudo 503 CDs que estavam com o endereço de e-mail atualizado, naquele momento, na base de dados do Conselho Regional de Odontologia do Rio de Janeiro. As questões foram enviadas pelo programa de pesquisa por questionário (Survio). As questões respondidas pelos participantes do estudo encontram-se descritas nas Tabelas 1 , 2 e 3. Juntamente com o questionário, foi enviado o termo de consentimento livre e esclarecido, sendo que, ao responder ao questionário, o CD concordava automaticamente em participar do estudo.

Todos os dados foram mantidos em sigilo pelo grupo de pesquisadores e os clínicos participantes não têm acesso às informações dos demais participantes. Os dados obtidos foram analisados e apresentados como análise descritiva. Com o intuito de se entender 
qual a relação entre o perfil de formação e atendimento do $\mathrm{CD}$ e a forma pela qual conduz o diagnóstico e 0 tratamento das LCNCs, foi empregado o teste de Qui-Quadrado com nível de significância de 5\%.

\section{Resultados}

A Tabela 1 mostra as respostas referentes à formação e à forma de atendimento realizada pelos CDs. O questionário foi respondido por $119 \mathrm{CDs}$ (23,6\% do total da amostra), sendo $76(63,9 \%)$ do gênero feminino e $43(36,1 \%)$ do masculino. Quanto ao perfil de formação e atendimento dos CDs, $36(30,3 \%)$ apresentaram 21 ou mais anos de formado; $99(83,2 \%)$ cursaram especialização, sendo $24(20,2 \%)$ especialistas em Prótese Dentária; 65 $(54,6 \%)$ não atuam como clínico geral; e 69 (58\%) realizam atendimento particular.

Quanto ao perfil de diagnóstico das LCNCs (Tabela 2), os maiores valores observados foram de que $91(76,5 \%)$ respondentes revelaram fazer distinção entre as lesões de abfração, abrasão e erosão (biocorrosão), 99 (83,2\%) não fazem uso de nenhum método auxiliar no diagnóstico das LCNCs, 71 (59,7\%) não possuem nenhum questionário específico para diagnóstico de LCNCs. Ao serem questionados sobre a etiologia das LCNCs, $71(59,7 \%)$ atribuíram caráter multifatorial; $71(59,7 \%)$ nunca participaram de cursos e/ou palestras em que o tema abordado foi as LCNCs.

Quanto ao perfil do tratamento restaurador para as restaurações diretas de LCNCs (Tabela 3 ), os maiores valores observados foram de que 78 $(65,5 \%)$ utilizam isolamento relativo, $99(83,2 \%)$ fazem uso de técnicas de afastamento/retração gengival, $59(49,6 \%)$ usam apenas o fio afastador sem solução hemostática; 81 (68\%) realizam forramento com cimento ionômero de vidro (CIV) ou CIV modificado por resina, dependendo da profundidade, 62 $(52,1 \%)$ não realizam bisel em esmalte, 75 (63\%) utilizam sistema adesivo convencional de dois passos, $47(39,5 \%)$ dos CDs que utilizam adesivo autocondicionante fazem uso de ácido fosfórico em esmalte, 52 $(52,1 \%)$ utilizam resina híbrida/micro-híbrida para a restauração desse tipo de lesão, $112(94,1 \%)$ fazem uso da técnica incremental, 39 (32,8\%) informaram que as restaurações duram de três a cinco anos.

Os testes de Qui-Quadrado revelaram não haver associação entre o perfil do CD e a forma de diagnóstico (Tabela 4). As únicas relações estatisticamente significativas foram entre a forma de remuneração e o uso de isolamento $(p=0,004)$ (Tabela 5) e entre a forma de remuneração e o uso de técnicas de retração/afastamento gengival $(\mathrm{p}=0,005)$ (Tabela 6).
Tabela 1 - Perfil de formação e atendimento dos CDs

\begin{tabular}{|c|c|c|}
\hline & \\
\hline & $\mathrm{N}$ & $\%$ \\
\hline Total de avaliados & 119 & 100 \\
\hline \multicolumn{3}{|l|}{ Gênero } \\
\hline Masculino & 43 & 36,1 \\
\hline Feminino & 76 & 63,9 \\
\hline \multicolumn{3}{|c|}{ Anos desde o término da graduação em Odontologia } \\
\hline$<5$ anos & 14 & 11,8 \\
\hline 5 a 10 anos & 32 & 26,9 \\
\hline 11 a 15 anos & 26 & 21,8 \\
\hline 16 a 20 anos & 11 & 9,2 \\
\hline 21 ou mais anos & 36 & 30,3 \\
\hline \multicolumn{3}{|c|}{ Especialista/especialidade } \\
\hline Dentística & 21 & 17,6 \\
\hline Periodontia & 9 & 7,6 \\
\hline Prótese dentária & 24 & 20,2 \\
\hline Outra & 45 & 37,8 \\
\hline Não & 20 & 16,8 \\
\hline \multicolumn{3}{|c|}{ Atua como clínico(a) geral? } \\
\hline Sim & 54 & 45,4 \\
\hline Não & 65 & 54,6 \\
\hline
\end{tabular}

Com relação à população de pacientes atendidos, qual a forma de remuneração predominante?

\begin{tabular}{l|c|c|}
\hline Atendimento de plano odontológico & 20 & 16,8 \\
Atendimento de serviço público & 30 & 25,2 \\
Atendimento particular & 69 & 58 \\
\hline
\end{tabular}

Fonte: dados da pesquisa.

Tabela 2 - Perfil de diagnóstico das LCNCs

\section{\begin{tabular}{l|l}
$\mathrm{N}$ & $\%$
\end{tabular}}

Em casos de LCNCs, você procura fazer distinção entre lesões de abfração, abrasão e/ou erosão (biocorrosão)?

\begin{tabular}{l|c|c}
\hline Sim & 91 & 76,5 \\
Não & 28 & 23,5 \\
\hline
\end{tabular}

Faz uso de algum método auxiliar para diagnóstico de LCNCs?

\begin{tabular}{l|c|c} 
Sim & 20 & 16,8
\end{tabular}

\begin{tabular}{l|l|l} 
Não & 99 & 83,2
\end{tabular}

Possui um questionário específico para diagnóstico de LCNCs?

\begin{tabular}{l|c|c} 
Sim & 48 & 40,3
\end{tabular}

\begin{tabular}{l|l|l} 
Não & 71 & 59,7 \\
\hline
\end{tabular}

\begin{tabular}{|c|c|c|}
\hline \multicolumn{3}{|c|}{ Etiologia das LCNCs? } \\
\hline Unifatorial & 27 & 22,7 \\
\hline Multifatorial & 71 & 59,7 \\
\hline Não sei responder & 21 & 17,6 \\
\hline \multicolumn{3}{|c|}{$\begin{array}{l}\text { Já participou de algum curso e ou palestra em que o tem } \\
\text { abordado tenha sido LCNCs? }\end{array}$} \\
\hline Sim & 48 & 40,3 \\
\hline Não & 71 & 59,7 \\
\hline
\end{tabular}

Fonte: dados da pesquisa. 
Tabela 3 - Perfil do tratamento restaurador para restaurações diretas de LCNCS

\begin{tabular}{|c|c|c|}
\hline & \\
\hline & $\mathrm{N}$ & $\%$ \\
\hline \multicolumn{3}{|c|}{ Para o tratamento restaurador de LCNCs, faz o uso de isolamento? } \\
\hline Não, sem isolamento & 16 & 13,4 \\
\hline Sim, isolamento absoluto & 25 & 21 \\
\hline Sim, isolamento relativo & 78 & 65,6 \\
\hline \multicolumn{3}{|c|}{ Para o tratamento restaurador de LCNCs, faz o uso de técnicas de retração/afastamento gengival? } \\
\hline Sim & 99 & 83,2 \\
\hline Não & 20 & 16,8 \\
\hline \multicolumn{3}{|c|}{ Em caso do uso do fio afastador, utiliza substância hemostática? } \\
\hline Nunca uso o fio afastador & 31 & 26 \\
\hline Sim & 29 & 24,4 \\
\hline Não & 59 & 49,6 \\
\hline \multicolumn{3}{|l|}{ Realiza forramento com CIV ou CIV modificado? } \\
\hline Sim, sempre & 7 & 6 \\
\hline Sim, dependendo da profundidade & 81 & 68 \\
\hline Nunca & 31 & 26 \\
\hline \multicolumn{3}{|l|}{ Realiza bisel em esmalte? } \\
\hline Sim & 57 & 47,9 \\
\hline Não & 62 & 52,1 \\
\hline \multicolumn{3}{|c|}{ Qual o sistema adesivo mais utilizado nas restaurações de LCNCs? } \\
\hline $\begin{array}{l}\text { Convencional de três passos } \\
\text { (Ex.: Scotchbond Multi-Uso, Opti-Bond) }\end{array}$ & Convencional de três passos & 22,7 \\
\hline \multicolumn{3}{|l|}{ Convencional de dois passos } \\
\hline (Ex.: Single-Bond, Prime \& Bond) & 75 & 63 \\
\hline \multicolumn{3}{|l|}{ Autocondicionante de dois passos } \\
\hline (Ex.: Clearfill SE Bond, Adper SE Plus) & 12 & 10,1 \\
\hline \multicolumn{3}{|l|}{ Autocondicionante de passo único } \\
\hline (Ex.: IBond, Adper Easy One) & 4 & 3,4 \\
\hline Não sei responder & 1 & 0,8 \\
\hline \multicolumn{3}{|c|}{ Caso utilize adesivo autocondicionante, faz uso de ácido fosfórico em esmalte? } \\
\hline Sim & 47 & 39,5 \\
\hline Não & 8 & 6,7 \\
\hline Não uso adesivo autocondicionante & 64 & 53,8 \\
\hline \multicolumn{3}{|c|}{ Caso utilize, qual a resina composta que você mais emprega para as restaurações de LCNCs? } \\
\hline \multicolumn{3}{|c|}{\begin{tabular}{|l|l} 
Híbrido/Microhíbrido &
\end{tabular}} \\
\hline \multicolumn{3}{|l|}{ Microparticulado } \\
\hline (Ex: Durarafill VS, Renamel, Heliofill) & 14 & 11,8 \\
\hline \multicolumn{3}{|l|}{ Nanoparticulado } \\
\hline (Ex: Filtek Supreme, Z350, Z350XT, Estelite) & 37 & 31,1 \\
\hline Não sei responder & 5 & 4,2 \\
\hline Não utilizo resina composta & 1 & 0,8 \\
\hline \multicolumn{3}{|l|}{ Faz uso da técnica incremental? } \\
\hline $\operatorname{Sim}$ & 112 & 94,1 \\
\hline Não & 6 & 5 \\
\hline Não uso resina para restaurar LCNCs & 1 & 0,8 \\
\hline
\end{tabular}

\begin{tabular}{|c|c|c|}
\hline \multicolumn{3}{|c|}{$\begin{array}{l}\text { Com base em seus conhecimentos e nas suas experiências clínicas, de maneira geral, qual o tempo de duração de uma restauração } \\
\text { de LCNCs? }\end{array}$} \\
\hline$<1$ ano & 4 & 3,4 \\
\hline 1 ano & 8 & 6,7 \\
\hline 2 anos & 27 & 22,7 \\
\hline 3 anos & 20 & 16,8 \\
\hline 3 a 5 anos & 39 & 32,8 \\
\hline 6-a 10 anos & 20 & 16,8 \\
\hline 11 a 15 anos & 1 & 0,8 \\
\hline
\end{tabular}

Fonte: dados da pesquisa. 
Tabela 4 - Valores de significância encontrados para o teste de Qui-Quadrado

\begin{tabular}{c|c|c}
\hline Anos desde & Atua & Com relação à população \\
o término da & como & de pacientes atendidos, qual \\
graduação em & clínico(a) & a forma de remuneração \\
Odontologia & geral? & predominante? \\
\hline
\end{tabular}

Perfil versus diagnóstico

Em caso de LCNCs, você procura fazer distinção entre lesões de abfração, abrasão e/ou erosão (biocorrosão)?

Faz uso de algum método auxiliar para diagnóstico de LCNCs?

0,451

0,603

0,109

Possui um questionário específico para diagnóstico de LCNCs?

Etiologia das LCNCs?

Perfil versus tratamento restaurador

Para o tratamento restaurador de LCNCs, faz o uso de isolamento?

Para o tratamento restaurador de LCNCs, faz o uso de técnicas de retração/afastamento gengival?

Em caso do uso do fio afastador, utiliza substância hemostática?

Realiza forramento com CIV ou CIV modificado por resina?

Realiza bisel em esmalte?

Qual o sistema adesivo mais utilizado nas restaurações de LCNCs?

Caso utilize adesivo autocondicionante, faz uso de ácido fosfórico em esmalte?

Caso utilize, qual a resina composta que você mais emprega para as restaurações de LCNCs?

Faz uso da técnica incremental?

Com base em seus conhecimentos e nas suas experiências clínicas, de maneira geral, qual o tempo de duração de uma restauração de LCNCs?

Fonte: dados da pesquisa.

Tabela 5 - Distribuição dos resultados obtidos para o teste de associação entre forma de remuneração e uso/forma de isolamento do elemento dental $(p=0,004)$

\begin{tabular}{|c|c|c|c|c|c|}
\hline & & \multicolumn{3}{|c|}{$\begin{array}{l}\text { Para o tratamento restaurador de LCNCs, faz o uso } \\
\text { de isolamento? }\end{array}$} & \multirow{2}{*}{ Total } \\
\hline & & $\begin{array}{l}\text { Não, sem } \\
\text { isolamento }\end{array}$ & $\begin{array}{l}\text { Sim, isolamento } \\
\text { absoluto }\end{array}$ & $\begin{array}{l}\text { Sim, isolamento } \\
\text { relativo }\end{array}$ & \\
\hline \multirow{3}{*}{$\begin{array}{l}\text { Com relação à população } \\
\text { de pacientes atendidos, } \\
\text { qual a forma de remune- } \\
\text { ração predominante? }\end{array}$} & Atendimento de serviço público & 7 & 8 & 15 & 30 \\
\hline & Atendimento particular & 3 & 16 & 50 & 69 \\
\hline & Atendimento de plano odontológico & 6 & 1 & 13 & 20 \\
\hline \multicolumn{2}{|l|}{ Total } & 16 & 25 & 78 & 119 \\
\hline
\end{tabular}

Fonte: dados da pesquisa.

Tabela 6 - Distribuição dos resultados obtidos para o teste de associação entre forma de remuneração e uso de técnicas de retração/afastamento gengival $(p=0,005)$

\begin{tabular}{|c|c|c|c|c|}
\hline & & \multicolumn{2}{|c|}{$\begin{array}{l}\text { Para o tratamento restaurador de LCNCs, faz o } \\
\text { uso de técnicas de retração/afastamento gengival? }\end{array}$} & \multirow{2}{*}{ Total } \\
\hline & & Não & Sim & \\
\hline \multirow{3}{*}{$\begin{array}{l}\text { Com relação à população de } \\
\text { pacientes atendidos, qual a } \\
\text { forma de remuneração pre- } \\
\text { dominante? }\end{array}$} & Atendimento de serviço público & 9 & 21 & 30 \\
\hline & Atendimento particular & 5 & 64 & 69 \\
\hline & Atendimento de plano odontológico & 6 & 14 & 20 \\
\hline \multicolumn{2}{|l|}{ Total } & 20 & 99 & 119 \\
\hline
\end{tabular}




\section{Discussão}

Diante da necessidade de melhor compreensão do comportamento dos CDs em relação aos processos de diagnóstico e tratamento restaurador de LCNCs e com a intenção de criar uma rede colaborativa em odontologia, o presente estudo representa uma primeira tentativa de interação com os clínicos registrados no Conselho Regional de Odontologia do Rio de Janeiro (CRO/RJ). Desse modo, a amostra contemplou os CDs que estavam com o cadastro atualizado na base de dados do conselho e com o endereço de e-mail disponível. Sabe-se que a quantidade de CDs registrados no $\mathrm{CRO} / \mathrm{RJ}$ é muito superior aos $503 \mathrm{CDs}$ que estavam com o e-mail atualizado na base de dados. Assim, inclusive, visando facilitar e melhorar a comunicação com os dentistas, o $\mathrm{CRO} / \mathrm{RJ}$ iniciou uma campanha para o recadastramento por meio eletrônico, processo que se encontra em andamento. Também é importante salientar a iniciativa do $\mathrm{CRO} / \mathrm{RJ}$ em estimular a prática baseada em evidências providas por redes colaborativas. Para tanto foi criada uma comissão específica para desenvolver estudos nesse formato, que surgiu após a realização do presente estudo, e verificar a necessidade de estimular os clínicos a participar dessa modalidade de estudo e buscar aprimoramento profissional.

Um aspecto interessante é que, apesar de 76,5\% dos participantes do estudo relatarem distinguir as lesões de abfração, abrasão e erosão (biocorrosão), a grande maioria $(83,2 \%)$ não emprega um método auxiliar para diagnóstico. Além disso, apesar dos diversos estudos realizados na área, demostrando que a etiologia das LCNCs é de origem multifatorial ${ }^{6,8,10,18,19}$, um número considerável de $\mathrm{CDs}$ $(40,3 \%)$, contrariando a literatura atual, não soube classificar as lesões quanto à etiologia ou atribuíram às lesões um caráter unifatorial, o que pode induzir a um diagnóstico incorreto e a uma escolha de tratamento muitas vezes falho, sem a eliminação dos fatores causais. Tais divergências podem estar relacionadas à formação acadêmica em odontologia, que, de maneira geral, não aplica um protocolo de diagnóstico direcionado para a determinação dos possíveis fatores etiológicos, a diferenciação e o tratamento dessas lesões ${ }^{17}$.

Dessa forma, pode-se sugerir a elaboração de um protocolo voltado para o diagnóstico, a diferenciação e o tratamento corretos das LCNCs, que seja prático e de fácil execução, podendo ser aplicado na formação acadêmica dentro das instituições de ensino superior e replicado na prática clínica diária. Tendo em vista que o tempo de formado não foi associado ao uso de formas específicas de diagnóstico para LCNCs, mesmo nos dias atuais, a formação acadêmica básica em odontologia é deficitária em relação ao tema e necessita de uma revisão urgente.
Quanto à forma de isolar ou não isolar o dente a receber o procedimento restaurador das lesões, o isolamento relativo foi o que obteve maior porcentagem de respostas $(65,6 \%)$, o que está diretamente relacionado a pré-molares/face vestibular, descrito como o grupo de dentes mais acometido por essas lesões ${ }^{20}$. Essa região possibilita um bom controle da umidade, boa visualização da área a ser restaurada, além da praticidade e do tempo de execução da técnica.

Um dado que mostrou significância estatística e que deve ser levado em consideração é a forma de utilização, ou não, de isolamento $(\mathrm{p}=0,004)$ e as técnicas de retração/afastamento gengival ( $p=0,005$ ), quando correlacionados com a população de pacientes atendidos, o que sugere que essas técnicas estão vinculadas, principalmente, ao tratamento de pacientes de atendimento particular. Esse dado mostra que o comportamento do CD pode estar associado às condições de trabalho em que se encontra quando empregado e sobre as quais nem sempre exerce influência de forma direta e imediata, como no setor público, e também pela forma de remuneração. Tal achado é preocupante, tendo em vista que a escolha de um material ou de uma técnica deve ser voltada para o melhor resultado a ser obtido para o paciente e deve ser usado como alerta na formação dos CDs, seja de graduação ou pós-graduação.

Assim, esse formato de estudo, que visa ao entendimento sobre os fatores que influenciam as escolhas e decisões na prática clínica, deve ser encorajado. No caso dos estudos em rede colaborativa, existe o benefício de retorno das informações aos CDs, que devem ser alertados com relação a algumas atitudes na prática clínica. Outros estudos revelam que o tipo de prática e a forma de remuneração podem influenciar diversas decisões clínicas, como o limite para a intervenção restauradora no tratamento de lesões de cárie ${ }^{25,27}$.

Para que se possa obter uma restauração de compósito de alto desempenho, especialmente em lesões de LCNCs, é necessário um bom isolamento da margem gengival a fim de evitar a contaminação por um provável sangramento gengival e/ou pelo fluido cervicular ${ }^{28}$. Assim, agentes hemostáticos são utilizados com certa frequência, sendo que, no presente estudo, $33 \%$ dos CDs que utilizam fio retrator também usam agentes hemostáticos. A maior parte dos agentes hemostáticos apresenta propriedades ácidas $(\mathrm{pH}=0,7-3)$ e características hidrófilas que podem contaminar e interferir na restauração adesiva $^{29}$. É importante salientar que os fabricantes preconizam a realização de um enxague prévio ao procedimento adesivo, a fim de eliminar possíveis contaminações da cavidade a ser restaurada, tornando praticamente nulo o efeito do agente hemostático sobre o procedimento adesivo ${ }^{30}$.

Neste estudo, 68\% dos CDs utilizam, dependendo da profundidade da lesão, os cimentos de ionômero de vidro e os cimentos de ionômero de vidro modificados por resina para base ou forramento da 
cavidade. Esses cimentos são utilizados em diversas situações na prática odontológica, principalmente para as restaurações de LCNCs, devido à sua capacidade de aderir ao esmalte e à dentina e à sua capacidade de liberação de flúor para o meio bucal, que confere propriedade anticariogênica ao material $^{31}$. Diversos estudos in vitro e in situ demonstram vantagens do uso de materiais contendo flúor na progressão de lesões de cárie. De outro modo, o efeito anticariogênico de materiais liberadores de flúor não está baseado em evidência clínica e, além disso, pode ser substituído pelos fluoretos liberados pelos dentifrícios ${ }^{32}$.

Não existe, na literatura, evidência científica conclusiva a respeito do uso ou não do bisel em restaurações na região cervical. Observa-se que no presente estudo 47,9\% dos CDs realizam tal procedimento. A técnica visa basicamente aumentar a área de exposição dos prismas de esmalte com o intuito de aumentar a retenção da restauração e mascarar a interface entre o esmalte e o composto a fim de melhorar a estética. A única desvantagem descrita desse procedimento é que há a necessidade de desgaste de uma pequena quantidade de estrutura dental $^{33}$. Cavidades preparadas com bisel em esmalte apresentam comportamento semelhante ao das que não foram biseladas, mas proporcionam melhor vedamento marginal, diminuindo a microinfiltração ${ }^{34}$.

Um dos maiores desafios da odontologia restauradora diz respeito aos processos de adesão ao esmalte e à dentina. Enquanto a adesão ao esmalte apresenta-se de forma previsível e eficaz do ponto de vista científico e clínico, devido ao seu substrato ser basicamente homogêneo (96\% inorgânico), a adesão em dentina imprime um grande desafio aos clínicos e pesquisadores devido à grande presença de material orgânico (20\%) e água (10\%), que deixa o substrato heterogêneo e a técnica de adesão em dentina altamente sensível ${ }^{35}$.

Sabe-se que os sistemas adesivos mais indicados para as restaurações das LCNCs são os convencionais de três passos, devido à capacidade de formar uma camada híbrida consistente, e os autocondicionantes de dois passos, devido à menor sensibilidade da técnica, à menor sensibilidade pós-operatória e à adesão química na estrutura dental, proporcionada pela adição do monômero metacriloiloxidecil diidrogenofosfato (10-MDP) no sistema adesivo ${ }^{36-41}$.

Entretanto, no presente estudo, a maioria dos CDs (63\%) utiliza o sistema adesivo convencional de dois passos, apesar de esses sistemas adesivos induzirem a piores resultados quanto à durabilidade das ligações adesivas ${ }^{42}$. Esse fato pode estar relacionado diretamente com as falhas nos procedimentos de adesão ao substrato dentinário, tendo em vista que a discrepância entre a área desmineralizada e a área infiltrada pelo agente de união é uma das principais causas de falhas nesse processo de adesão.

Dessa forma, é importante que os profissionais envolvidos na formação acadêmica e em educação continuada atuem de maneira mais eficiente na translação do conhecimento científico para os clínicos e que esses, por sua vez, busquem uma constante atualização de conhecimento. Ainda em relação aos adesivos, 85,5\% dos CDs que utilizam adesivo autocondicionante realizam condicionamento ácido em esmalte, o que deve ficar claro é que esse procedimento não apresenta qualquer prejuízo à técnica adesiva $^{43}$.

Com base em estudos clínicos de longo prazo e ensaios laboratoriais, a resina composta é o material de escolha para as restaurações de LCNCs, devido às suas propriedades físicas, capacidade de adesão ao sistema adesivo, excelente estética, boa resistência ao desgaste, facilidade de manipulação e boa relação custo-beneficio ${ }^{44}$. Neste estudo, a grande maioria dos $\operatorname{CDs}(52,1 \%)$ relatou utilizar resina composta híbrida/micro-híbrida, mas é importante ressaltar que não há dado algum na literatura que suporte qual o melhor compósito para esse tipo de restauração.

Quando perguntados sobre a utilização da técnica incremental para inserção da resina composta na área a ser restaurada, 94,1\% dos CDs responderam fazer uso da técnica incremental, provavelmente embasados no fato de que tal técnica diminui as tensões de polimerização e, em teoria, reduz a formação de fendas marginais ${ }^{45}$. A formulação dos compósitos apresenta pouca influência em restaurações classe $\mathrm{V}$ devido a um pequeno fator-C, enquanto que o sucesso da restauração está diretamente relacionado ao sistema adesivo empregado ${ }^{36}$.

Com relação à longevidade das restaurações de LCNCs, apenas $17,6 \%$ dos CDs acreditam na duração por um período acima de cinco anos. Como descrito pela literatura atual, o sucesso dessas restaurações está intimamente relacionado com o sistema adesivo utilizado. Estudos clínicos de acompanhamento de longo prazo demonstraram que sistemas adesivos convencionais de três passos apresentam taxa de retenção de $94 \%$ em acompanhamento clínico de treze anos, assim como os sistemas adesivos autocondicionantes de dois passos, que apresentam taxa de retenção de $97 \%$ durante oito anos de acompanhamento clínico ${ }^{36,41}$.

Assim, pode-se supor que a impressão de baixa durabilidade de restaurações em LCNCs descrita pela maioria dos entrevistados do presente estudo esteja associada ao uso de sistemas adesivos convencionais de dois passos, empregado pela maioria dos CDs participantes do estudo. Além disso, pode-se questionar a capacidade dos CDs em determinar a real necessidade de substituição de restaurações realizadas com resinas compostas. Infelizmente, tópicos envolvendo os critérios de substituição de restaurações são pouco abordados na formação dos CDs. Portanto, novos estudos que envolvam os critérios de substituição de restaurações estão planejados.

O presente estudo teve como base uma primeira tentativa de formação de rede de pesquisa colabo- 
rativa no estado do Rio de Janeiro. Igualmente, é importante salientar que a partir dele foram desenvolvidas novas ações, como a Comissão de Estudos e Ações em Rede de Pesquisa Odontológica Baseada em Evidências, que tem estimulado a participação dos CDs no processo de constante troca de informações entre clínicos e acadêmicos.

\section{Conclusões}

Com base nos resultados obtidos, conclui-se que:

1. Os CDs entrevistados não possuem um protocolo preestabelecido para correto diagnóstico de LCNCs.

2. A forma de remuneração influencia no uso do isolamento e nas técnicas de retração/afastamento gengival para o tratamento restaurador.

3. O sistema adesivo convencional de dois passos é o mais utilizado pelos CDs.

Com o aumento da incidência das LCNCs devido ao envelhecimento da população e à exposição aos prováveis fatores etiológicos, novos estudos clínicos e laboratoriais são necessários, com o intuito de descrever especificamente qual a etiologia dessas lesões bem como determinar quais técnicas e materiais restauradores apresentam melhores resultados em longo prazo.

\section{Agradecimentos}

Os autores agradecem o apoio do Conselho de Odontologia do Estado do Rio de Janeiro (CRO-RJ), ao $\mathrm{CNPq}$ (Pibic/UFF) e da Fundação de Amparo à Pesquisa do Estado do Rio de Janeiro (Faperj) na modalidade Jovem Cientista do Nosso Estado.

\section{Abstract}

Objective: To identify the profile of training and care of dental-surgeons (DDSs), attempting to understand what is the conduct provided by DDSs in terms of diagnosis and treatment of non-carious cervical lesions (NCCLs). Subjects and method: A questionnaire was offered and sent to DDSs with updated records in the database of the Regional Council of Dentistry of Rio de Janeiro (CRO- RJ) - Brazil. The results were analyzed descriptively and the chi-square test was performed at $5 \%$ significance level. Results: Most DDSs seeks to distinguish the different forms of NCCLs (76.5\%), but does not use an auxiliary method (83.2\%) and has no specific questionnaire (59.7\%). To $40.3 \%$ of DDSs, the NCCL etiology is unifactorial or they could not answer this question. Most of them uses relative isolation (65.6\%) and gingival retraction/separation techniques $(83.2 \%)$. The conventional two-step adhesive system (63\%) and hybrid/microhybrid composite resin (52.1\%) are the most commonly used materials. Regarding the longevity of restorations, only $17.6 \%$ of DDSs believes they last over 5 years. Regarding associations, there was a relationship between the form of remuneration and the use/method of isolation $(p=0.004)$, and between the form of remuneration and the use of gingival retraction/separation techniques $(p=0.005)$. Conclusions: The DDSs do not have a pre-established protocol for correct diagnosis of NCCLs. The form of remuneration influences the use of isolation for restorative treatment. The conventional two-step adhesive system is mostly used by DDSs.

Keywords: Abfraction. Abrasion. Biocorrosion. Erosion. Non-carious cervical lesions.

\section{Referências}

1. Hattab FN, Yassin OM. Etiology and diagnosis of tooth wear: a literature review and presentation of selected cases. Int $\mathrm{J}$ Phosthod 2000; 13(2):101-7.

2. Bader JD, Mcclure F, Scurria MS, Shugars DA, Heymann HO. Case-control study of non-carious cervical lesions. Community Dent Oral Epidemiol 1996; 24(4):286-91.

3. Kliemann C. Lesões cervicais não cariosas por abrasão (escovação traumática). JBC J Bras Clin Estet Odontol 2002; 6(33):204-9.

4. Bader JD, Levitch LC, Shugars DA, Heymann HO, Mcclure, F. How dentist classified and treated non-carious cervical lesions. J Am Dent Assoc 1993; 124(5):46-54.

5. AW TC, Lepe X, Jonhson GH, Mancl L. Characteristics of non-carious cervical lesions - a clinical investigation. J Am Dent Assoc 2002; 133(6):725-33.

6. Grippo JO, Simring M, Schreiner S. Attrition, abrasion, corrosion and abfraction revisted. J Am Dent Assoc 2004; 135:1109-18

7. Levitch LC, Bader JD, Shugars DA, Heymann HO. Non-carious cervical lesions. J Dent 1994; 22(4):195-207.

8. Bernhardt O, Gesch D, Schwahn C, Mack F, Meyer G, John $\mathrm{U}$, et al. Epidemiological evaluation of the multifactorial aetiology of abfractions. J Oral Rehabil 2006; 33(1):17-25.

9. Grippo JO, Simring M. Dental erosion revisted. J Am Dent Assoc 1995; 126(5):619-30.

10. Grippo JO, Coleman TA, Simring M. Abfraction, abrasion, biocorrosion, and the enigma of noncarious cervical lesions: a 20-year perspective. J Esthet Restor Dent 2012; 24(1):10-23.

11. Grippo JO. Abfractions: a new classification of hard tissue lesions of teeth. J Esthet Dent 1991; 3(1):14-9.

12. Osborne-Smith KL, Burke FJ, Wilson NH. The aetiology of the non-carious cervical lesions. Int Dent J 1999; 49(5):139-43.

13. Grippo JO, Masi JV. Role of Biodental Engineering Factors (BEF) in the etiology of root caries. J Esthet Dent 1991; 3(2):71-6.

14. Lee WC, Eakle S. Possible role of tensile stress in the etiology of cervical erosive lesions of teeth. J Prosth Dent 1984; 52(3):374-80

15. Bartlett DW. The role of erosion in tooth wear: aetiology, prevention and management. In Dent J 2005; 55(4 Suppl 1):277-84.

16. Lee WC, Eakle WS. Stress-induced cervical lesions: review of advances in the past 10 years. J Prosthet Dent 1996; 75(5):487-94.

17. Santos-Daroz CB, Daroz LGD, Batitucci MHG, Batitucci G, Miranda MS. Classification and treatment of non-carious dental lesions by under-graduate dental students. UFES Rev Odontol 2007; 9(1):5-12. 
18. Braem M, Lambretchs P, Vanherle G. Stress-induced cervical lesions. J Prosthet Dent 1992; 67(5):718-22.

19. Addy M, Hunter ML. Can tooth brushing damage your health? Effects on oral and dental tissues. Int Dent J 2003; 53(Suppl 3):177-86.

20. Santos FFC, Lopes FF, Thomaz EBAF, Benatti BB, Pereira AFV. Evaluation of non-carious cervival lesions in adults: a pilot study. Pesq Bras Odontoped Clin Integr 2013; 13(1):31-6.

21. Lyttle HA, Sidhu N, Smyth B. A study of the classification and treatment of non carious cervical lesions by general practitioners. J Prosth Dent 1998; 79(3):342-6.

22. Gilbert GH, Williams D, Rindal B, Pihlstrom DJ, Benjamil PL, Wallace MC. The creation and development of the Dental Practice-Based Research Network. J Am Dent Assoc 2008; 139(1):74-81.

23. Giannobile WV. Dentistry, oral health, and clinical investigation. J Dent Res 2012; 91:S3.

24. Gordan VV. Translating research into everyday clinical practice: Lessons learned from a USA dental practice-based research network. Dent Mater 2012; 29(1):3-9.

25. Kakudate N, Sumida F, Manabe K, Yokohama Y, Gilbert GH, Goran VV. Restorative treatment thresholds for proximal caries in dental PBRN. J Dent Res 2012; 91(12):1202-8.

26. American Dental Association. Policy on evidence-based dentistry: definition of evidence-based dentistry. Chicago: ADA; 2008.

27. Gordan VV, Garvan CW, Heft MW, Fellows JL, Qvist V, Rindal DB. Restorative treatment thresholds for interproximal primary caries based on radiographic images: findings from the Dental Practice-Based Research Network. Gen Dent 2009; 57:654-63.

28. Kaneshima T, Yatani H, Kasai T, Watanabe EK, Yamashita A. The influence of blood contamination on bond strengths between dentin and an adhesive resin cement. Oper Dent 2000; 25:195-201

29. Cenci MS, Pereira-Cenci T, Donassollo TA, Sommer L, Strapasson A, Demarco FF. Influence of thermal stress on marginal integrity of restorative materials. J Appl Oral Sci 2008; 16:106-10.

30. Ebrahimi SF, Shadman N, Abrishami A. Effect of ferric sulfate contamination on the bonding effectiveness of etch-andrinse and self-etch adhesives to superficial dentin. J Conserv Dent 2013; 16(2):126-30.

31. Van Noort R. Introdução aos materiais dentários. 3. ed. Rio de Janeiro: Elsevier; 2010. p. 119-33.

32. Cury JA, Oliveira BH, Santos AP, Tenuta LM. Are fluoride releasing dental materials clinically effective on caries control? Dent Mater 2016; 32:323-33.

33. Coelho-de-Souza FH, Camacho GB, Demarco FF, Powers J. Fracture resistance and gap formation of MOD restoration: influence of restorative technique, bevel preparation and water storage. Oper Dent 2008; 33:37-43.

34. Coelho-de-Souza FH, Camargo JC, Beskow T, Balestrin MD, Klein-Júnior CA, Demarco FF. A randomized doubleblind clinical trial of posterior composite restorations with or without bevel: 1-year follow-up. J Appl Oral Sci 2012; 20(2):174-9.

35. Frankenbergerf R, Kramer N, Petschelt A. Long-term effect of dentin primers on enamel bond strength and marginal adaptation. Oper Dent 2000; 25(1):11-9.

36. Van Meerbeek B, Peumans M, Poitevin A, Mine A, Van Ende A, Neves A, et al. Relationship between bond-strength test and clinical outcomes. Dent Mater 2010; 26(2):100-21.
37. Van Meerbeek B, Yoshihara K, Yoshida Y, Mine A, De Munck J, Van Landuyt KL. State of the art of self-etch adhesives. Dent Mater 2011; 27(1):17-28.

38. Moretto SG, Russo EMA, Carvalho RCR, De Munck J, Van Landuyt K, Peumans M, et al. 3-year clinical effectiveness of one-step adhesives in non-carious cervical lesions. Dent Mater 2013; 41(8):675-82.

39. Santos MJMC, Ari N, Steele S, Costella J, Banting D. Retention of tooth-colored restorations in non-carious cervical lesions - a systematic review. Clin Oral Invest 2014; 18:136981

40. Peumans M, De Munck J, Mine A, Van Meerbeek B. Clinical effectiveness of contemporary adhesives for the restoration of non-carious cervical lesions. A systematic review. Dent Mater 2014; 30(10):1089-103.

41. Peumans M, De Munck J, Van Landuyt K, Van Meerbeek B. Thirteen-year randomized controlled clinical trialof a twostep self-etch adhesive in non-carious cervical lesions. Dent Mater 2015; 31(3):308-14

42. Peumans M, Kanumilli P, De Munck J, Van Landuyt K, Lambrechts P, Van Meerbeek B. Clinical effectiveness of contemporary adhesives: a systematic review of current clinical trials. Dent Mater 2005; 21(9):864-81.

43. Watanabe T, Tsubota K, Takamizawa T, Kurokawa H, Rikuta $\mathrm{H}$, Ando S, et al. Effect of prior acid etching on bonding durability of single-step adhesives. Oper Dent 2008; 33(4):426-33.

44. Van Dijken JW. A randomized controlled 5-year prospective study of two HEMA-free adhesives, a 1-step self etching and a 3-step etch-and-rinse, in non-carious cervical lesions. Dent Mater 2013; 29(11):271-80.

45. Owens BM, Johnson WW. Effect of Insertion Technique and Adhesive System on Microleakage of Class V Resin Composite Restorations. J Adhes Dent 2005; 7(4):303-8.

\section{Endereço para correspondência:}

Luis Felipe Schneider

Núcleo de Pesquisa de Biomateriais Odontológicos (N-PBO)

Universidade Veiga de Almeida

20270-150 Rio de Janeiro, RJ, Brasil

Telefone: (21) 98102-0039

E-mail: felipefop@gmail.com

Recebido: 16/03/2016. Aceito: 15/09/2016. 